\title{
Development of criteria for a magnetic hydrocyclones
}

\author{
Boris Avdeyev ${ }^{1, *}$ \\ ${ }^{1}$ Kerch State Maritime Technological University, 298309 Kerch, Russia
}

\begin{abstract}
New criteria of a magnetic hydrocyclone - average value, magnetic field density and magnetic energy in a hydrocyclone are introduced in the article. Due to simplicity of a design and Ease of production and service, magnetic hydrocyclones have found broad application in virtually all sectors of industry, but more conspicuously advantages of their use reveal in purification systems of cooling lubricant while metal working. The dependence between field distribution in the working chamber and its average value hasn't been mathematically stated still. Complexity of criteria development and the determination of average value of the field is that the field strength changes depending on radius and height of the device. It is impossible to calculate the efficiency of cleaning from ferromagnetic impurity without knowledge of average value of magnetic field in the working chamber of a hydrocyclone therefore this problem is topical. The assumptions made in this paper are listed. Formulae are given, and numerical calculation of criteria of a hydrocyclone with radial magnetic field is shown by an example. Average value of the field, field density and magnetic energy in the working chamber of the device of inertial type has been defined on the basis of the dependence between field strength on the one hand both the radius and height of the field on the other hand which was obtained earlier.
\end{abstract}

\section{A problem statement}

Cooling lubricant which withdraw heat from workpiece, grease a surface, and delete residue are applied in the majority cases while metalworking [1-3]. Mechanical clearing of any technical liquid is carried out by one or various combination of four well-known methods: filtrational method, inertial method, gravitational method and separation (under the action of external fields). Devices of inertial type have found broad application in many industries and agriculture due to low cost, ease of manufacturing and maintenance, high efficiency, reliability and productivity [4-7].

Electric field is added to inertial force for the purpose of increase in efficiency of extraction of mechanical impurities [8]. A magnetic hydrocyclones which have begun to be used effectively since the fourth decade of XXth century can be referred to such devices. Devices vary not only in the geometrical dimensions, but also in an arrangement of sources of magnetic field, field parameters and other one (fig. 1) that demonstrates vigorous inventive work at combination of a magnetic and inertial method of clearing. Hydrocyclones with radial magnetic field, presented in fig. 2 a found the greatest use.

\section{Analysis of the last achievements and publications}

Magnetic hydrocyclones are fit for use if fast clearing of large amount of the cooling lubricant is required.
However, the field of application isn't limited to it: magnetic hydrocyclones can successfully be applied to extraction of ferromagnetic particles from the discharge liquor, to receive iron powders and iron-ore concentration, etc.

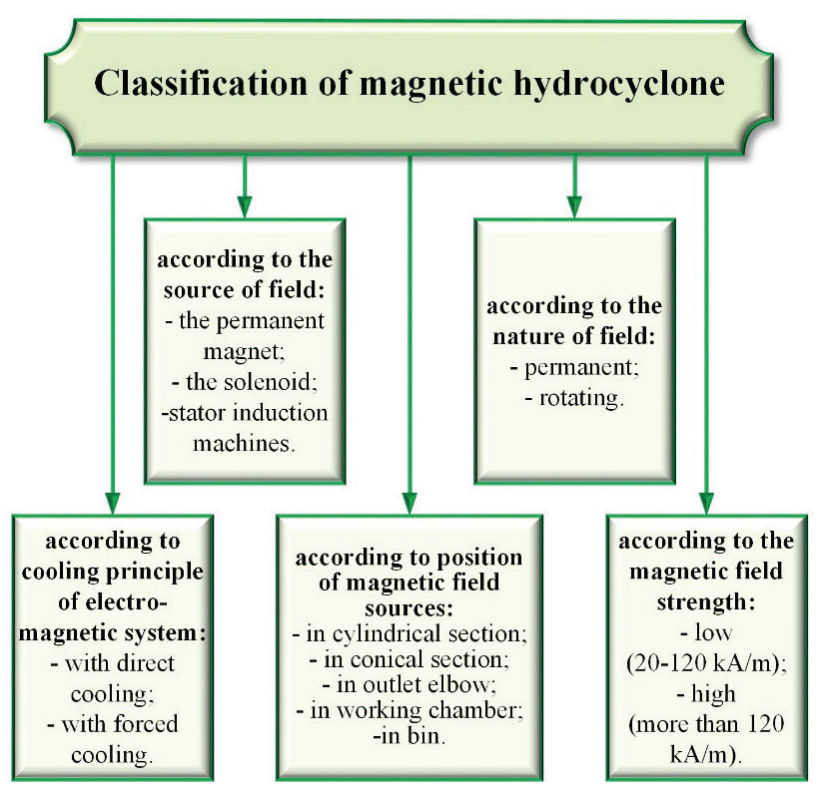

Fig. 1. Classification of magnetic hydrocyclones.

The most important parameters of devices with the superimposed magnetic field for clearing of viscous media from magnetic impurities are values of magnetic field in the working chamber and its configuration,

* Corresponding author: dirigeant@mail.ru 
necessary for calculation of forces of coagulation and the magnetic force affecting on a particle or a floccule in each point of the working chamber [9].

\section{Objectives and tasks of work}

The objective of this article is criteria development for evaluation of magnetic hydrocyclones on the basis of an evaluation of magnetic field in the working chamber of the device. The dependence between field distribution in the working chamber and its average value hasn't been mathematically stated still. The problem is in what in value of tension of the field changes depending on radius and height of the device. The criteria for evaluation of efficiency of the field given by J. Chen in [9] aren't acceptable in a consequence of need to know field strength in each point of space both complexity, and on occasion and impossibility of calculations of these criteria.

\section{The basic material of research}

The magnetic hydrocyclone (MHC) was developed as an outgrowth of a conventional hydrocyclone for the purpose of providing additional external magnetic force in addition to gravitational and centrifugal forces which cause grading and separation. Adding an external magnetic force, it is possible to increase the efficiently.

A vortex motion is being created in the separation space due to the tangential feed of the fluid being purified (fig $1 \mathrm{a}$ ). The liquid rotating at the outer wall of the apparatus goes down and in the center of apparatus goes upward. The down flow to the outer wall of the hydrocyclone is more important than gravity.

The particles are repelled to the wall of magnetic hydrocyclone under the action of centrifugal force, and they are transported to the bunker by the downward flow near the wall. The magnetic field is produced by a coil supplied by DC voltage, which is located between the main body and the vortex finder.

The particles coagulate with each other at the input of the apparatus. The magnetic field causes particles to attract each other, so the centrifugal force effecting the particles becomes stronger and operating efficiency rises.

The method of calculation of efficiency of a magnetic hydrocyclone is given in [8]. There is an average value of field strength among the parameters affecting efficiency of the device. Due to the fact that the field in the working chamber is inhomogeneous (fig. $2 \mathrm{~b}$ ), and is changed depending on radius and height of a hydrocyclone, it is advantageous to find such method of evaluation of its average value which adequately gives a true picture.

Magnetic field distribution was investigated in work [10]. The equations describing the change of magnetic field in the working chamber on radius and height of a hydrocyclone are given below:

$$
\left\{\begin{array}{l}
H(R, Z)=H_{1}^{\prime}(Z)+\left(H_{0}^{\prime}(Z)-H_{1}^{\prime}(Z)\right) \cdot e^{-\frac{\left(R-R_{0}\right)}{R_{1}-R_{0}} \cdot n} \\
H_{1}^{\prime}(Z)=H_{2}+\left(H_{1}-H_{2}\right) \cdot e^{-\frac{Z \cdot n}{Z_{1}}} \\
H_{0}^{\prime}(Z)=H_{3}+\left(H_{0}-H_{3}\right) \cdot e^{-\frac{Z \cdot n}{Z_{1}}}
\end{array}\right.
$$

where $\mathrm{H}_{0}-\mathrm{H}_{3}$ - magnetic field strengths, which are measured in the reference points (fig. 2), $\mathrm{A} / \mathrm{m} ; R_{0}$ - inner radius of hydrocyclone, $\mathrm{m} ; Z_{1}-$ the height of the cylinder part of hydrocyclone, $\mathrm{m} ; n-$ empirical coefficient, dimensionless quantity.

Since the field is radial, it is possible to neglect coordinate $\varphi[11,12]$. Actually the field won't be absolutely symmetric because the magnetic circuit is absent at the point of connection to the body of an inlet elbow and the field value will reach the maximum values at edges of a magnetic circuit. However, the field curvature has no significant effect on the general separation process therefore this phenomenon can be neglected [13-15].

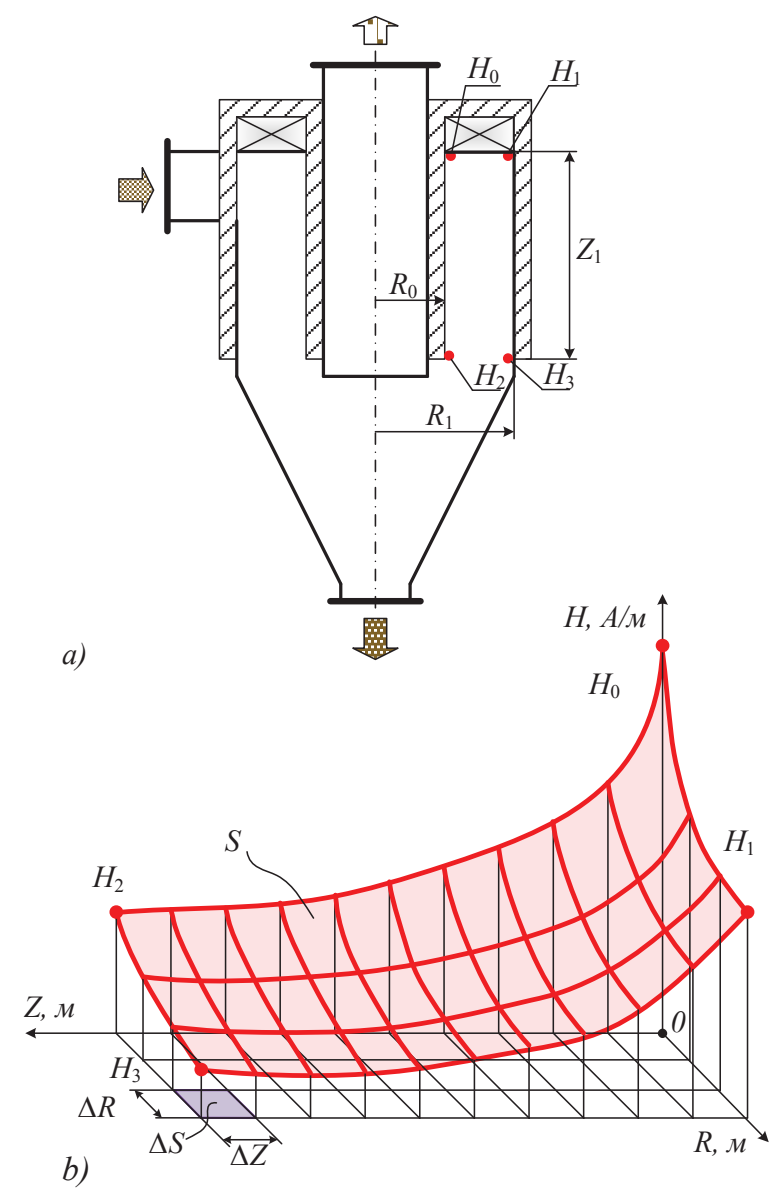

Fig. 2. Magnetic hydrocyclone (a) and changes in magnetic field strength in the working chamber of hydrocyclone (b)

Average value of fields at radial consideration can be calculated:

$$
\left\langle H_{S}\right\rangle=\frac{1}{S} \sum_{i} \sum_{j} H\left(R_{i}, X_{j}\right) \cdot \Delta R_{i} \cdot \Delta Z_{j},
$$


where $H\left(R_{i}, Z_{j}\right)$ - value of magnetic field strength in infinitesimal area $\Delta S_{i}$.

$$
S=\left(R_{1}-R_{0}\right) \cdot Z_{1} .
$$

The product of $H_{i}$ and $S_{i}$ in geometric sense represents value under the plane $S^{\prime}$.

Let's express (2) in the integrated form

$$
\left\langle H_{S}\right\rangle=\frac{1}{S} \iint_{S^{\prime}} H(R, Z) \mathrm{d} S^{\prime} .
$$

Expression (1) is cumbersome therefore let's use the simplified distribution formula of magnetic field which is measured both radially and throughout a height. A dependency with a high degree of precision represents real magnetic field in the working chamber of a hydrocyclone:

$$
H(R, Z)=\left[H_{3}+\left(H_{0}-H_{3}\right) \cdot e^{-\frac{Z \cdot n}{Z_{1}}}\right] \cdot\left(\frac{R_{0}}{R}\right)^{N} .
$$

Let's convert double integral (4) to iterated one, we get:

$$
\left\langle H_{S}\right\rangle=\frac{1}{S} \int_{0}^{Z_{1}} \mathrm{~d} Z \int_{R_{0}}^{R_{1}}\left[H_{3}+\left(H_{0}-H_{3}\right) \cdot e^{-\frac{Z \cdot n}{Z_{1}}}\right] \cdot\left(\frac{R_{0}}{R}\right)^{N} \mathrm{~d} R \cdot(
$$

Lowering calculations, $\left\langle H_{S}\right\rangle$ will take the following form:

$$
\begin{aligned}
\left\langle H_{S}\right\rangle= & \frac{1}{\left(R_{1}-R_{0}\right) \cdot Z_{1}} \cdot \frac{R_{0}^{N}}{N-1}\left(\frac{1}{R_{0}^{N-1}}-\frac{1}{R_{1}^{N-1}}\right) \times \\
& \times\left[H_{3} \cdot Z_{1}+\frac{Z_{1} \cdot\left(H_{3}-H_{0}\right)}{n}\left(1-e^{-n}\right)\right]
\end{aligned}
$$

For the case which is described in [model], average value of field strength is equal to $6,887 \mathrm{kA} / \mathrm{m}$ at the following values: $H_{0}=25 \mathrm{kA} / \mathrm{m} ; H_{3}=5 \mathrm{kA} / \mathrm{m} ; R_{0}=0,04$ $\mathrm{m} ; D=0,12 \mathrm{~m} ; Z_{1}=0,4 ; N=2,5 ; n=3$.

Average value of the field in the working chamber in cylindrical coordinate system can be written down:

$$
\left\langle H_{V}\right\rangle=\frac{1}{V} \sum_{i} \sum_{j} \sum_{k} H\left(R_{j}, \varphi_{i}, Z_{k}\right) \cdot R \cdot \Delta R_{i} \cdot \Delta \varphi_{j} \cdot \Delta Z_{k}
$$

where

$$
\Delta V=R \cdot \Delta R_{i} \cdot \Delta \varphi_{j} \cdot \Delta Z_{k}
$$

- volume element in cylindrical coordinate system (fig. 3).

Integrated form of equation will take a form:

$$
\left\langle H_{V}\right\rangle=\frac{1}{V} \iiint_{V} H(\varphi, R, Z) \mathrm{d} V .
$$

Let's convert triple integral (9), we get:

$$
\left\langle H_{V}\right\rangle=\frac{1}{V} \int_{R_{0}}^{R_{1}} R \mathrm{~d} R \int_{0}^{2 \pi} \mathrm{d} \varphi \int_{0}^{Z_{1}}\left[H_{3}+\left(H_{0}-H_{3}\right) e^{-\frac{Z \cdot n}{Z_{1}}}\right]\left(\frac{R_{0}}{R}\right)^{N} \mathrm{~d} Z
$$

$\left\langle H_{V}\right\rangle$ will take a form:

$$
\begin{aligned}
\left\langle H_{V}\right\rangle & =\frac{1}{\pi \cdot\left(R_{1}{ }^{2}-R_{0}{ }^{2}\right) \cdot Z_{1}} \cdot \frac{2 \pi \cdot R_{0}^{N}}{N-2}\left(\frac{1}{R_{0}^{N-2}}-\frac{1}{R_{1}^{N-2}}\right) \times \\
& \times\left[H_{3} \cdot Z_{1}+\frac{Z_{1} \cdot\left(H_{3}-H_{0}\right)}{n}\left(1-e^{-n}\right)\right]
\end{aligned}
$$

where

$$
V=\pi \cdot\left(R_{1}^{2}-R_{0}^{2}\right) \cdot Z_{1}
$$

- volume of working chamber of hydrocyclone, $\mathrm{m}^{3}$.

Average value of field strength $\left\langle H_{V}\right\rangle$ is equal to $6,045 \mathrm{kA} / \mathrm{m}$ at the same values, at which $\left\langle H_{S}\right\rangle$ was calculated. Relative error $\left\langle H_{S}\right\rangle$ with the respect to $\left\langle H_{V}\right\rangle$ is equal to $4,2 \%$, it is quite allowable.

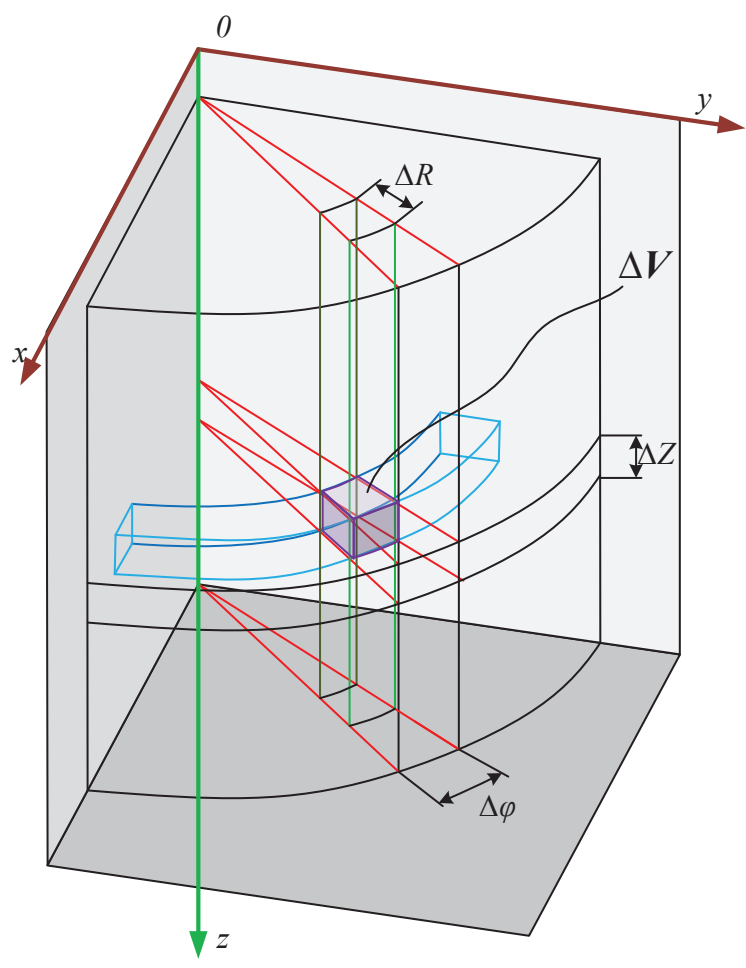

Fig. 3. Concerning calculating volume element $\Delta V$

The area density of magnetic field is calculated:

$$
\rho_{S}=\frac{\left\langle H_{S}\right\rangle}{S} .
$$

The volume density of a magnetic field:

$$
\rho_{V}=\frac{\left\langle H_{V}\right\rangle}{V} .
$$


Magnetic energy at volume $V$ is defined as follows:

$$
W_{M}=\frac{1}{2} \int_{V} H \cdot B \mathrm{~d} V,
$$

where $B$-magnetic induction, T.

Provided that

$$
B=\mu_{0} \cdot H,
$$

magnetic energy of field write down as:

$$
W_{M}=\frac{1}{2} \mu_{0} \int_{V} H^{2} \mathrm{~d} V,
$$

where $\mu_{0}$ - magnetic constant, $\mathrm{H} / \mathrm{m}$.

Let's substitute (5) and (9) into (18), we get:

$$
\begin{aligned}
W_{M} & =\frac{\pi \cdot R_{0}^{2 N}}{N-1}\left(\frac{1}{R_{0}^{2 N-2}}-\frac{1}{R_{1}^{2 N-2}}\right) \times \\
& \times\left[H_{3}^{2} \cdot Z_{1}+\frac{Z_{1}}{2 n}\left(H_{3}-H_{0}\right)\left(4 H_{3}+1\right)\left(1-e^{-2 n}\right)\right]
\end{aligned}
$$

Magnetic energy for magnetic hydrocyclone is equal $71,28 \mathrm{~kJ}$ to at the same values at which $\left\langle H_{S}\right\rangle$ was calculated.

\section{Conclusion}

Magnetic field of a hydrocyclone can be characterized by three values: average value of the field in the working chamber; volume density of magnetic field; magnetic energy. Average value of the field in the working chamber is necessary value for calculation of efficiency curve. Use of these criteria will allow to evaluate effect of magnetic field on motion of particle in working chambers of magnetic hydrocyclones.

\section{References}

1. Popov, A. Dugin, Int. J. Adv. Manuf. Technol. 76 (2015)

2. S. Chernyi, IOP Conf. Ser.: Mater. Sci. Eng. 124 (2017)

3. V.P. Astakhov, S. Joksch, Metalworking Fluids (MWFs) for Cutting and Grinding (Woodhead Publishing, 2012)

4. V. Budnik, S. Chernyi, Procedia Eng. 150 (2016)

5. V.A. Zhukov, E.P. Masyutkin, B.A. Avdeyev, IOP Conf. Ser.: Mater. Sci. Eng. 177 (2017)

6. L.-Y. Chu, W.-M. Chen, X.-Z. Lee, Sep. Purif. Technol. 26 (2002)

7. R.A. Augusto, J.R. Martins, Min. Proc. and Extr. Met. Rev. 22 (2001)

8. G. Chen, Design and analysis of magnetic hydrocyclone (M. Eng. Thesis, McGill University, Montreal, 1989)
9. B.A. Avdeyev Improving the efficiency of the engine oil cleaning in ship diesel engines through the use of magnetic hydrocyclones (Zebra, Ulyanovsk, 2016)

10. V.I. Prosvirnin, S.P. Golikov, B.A. Avdeyev, Bulletin of the Kherson National Technical University 46 (2013)

11. R.J. Freeman, N.A. Rowson, T.J. Veasey, I.R. Harris, IEEE Transactions on Magnetics 30 D (1994)

12. N. Nezami, B. Hajighorbani, International conference on sustainable development, strategies and challenges With a focus on Agriculture, Natural Resources, Environment and Tourism (2015)

13. J.R. Portela, J. Sánchez-Oneto, M. Belén García-Jarana, J. Martinez de la Ossa, Chem. Eng. Trans. 17 (2009)

14. R.H.M.G. Nabben, R.P.J. Duursma, A.A. Kamperman, J.L. Lagerberg, Ironmaking Steelmaking 25 (5) (1998)

15. A. Nyrkov, A. Shnurenko, S. Sokolov, S. Chernyi, \& V. Korotkov, Procedia Engineering 178 (2017). 